\title{
Improving breast cancer services for African-American women living in St. Louis
}

\author{
Lailea Noel $^{1} \cdot$ Shahnjayla K. Connors ${ }^{2} \cdot$ Melody S. Goodman $^{2} \cdot$ Sarah Gehlert $^{1,2}$ (I)
}

Received: 6 August 2015/Accepted: 22 September 2015/Published online: 26 September 2015

(C) The Author(s) 2015. This article is published with open access at Springerlink.com

\begin{abstract}
A mixed methods, community-based research study was conducted to understand how provider-level factors contribute to the African-American and white disparity in breast cancer mortality in a lower socioeconomic status area of North St. Louis. This study used mixed methods including: (1) secondary analysis of Missouri Cancer Registry data on all 885 African-American women diagnosed with breast cancer from 2000 to 2008 while living in the geographic area of focus; (2) qualitative interviews with a subset of these women; (3) analysis of data from electronic medical records of the women interviewed; and (4) focus group interviews with community residents, patient navigators, and other health care professionals. 565 women diagnosed with breast cancer from 2000 to 2008 in the geographic area were alive at the time of secondary data analysis; we interviewed $(n=96 ; 17 \%)$ of these women. Provider-level obstacles to completion of prescribed treatment included fragmented navigation (separate navigators at Federally Qualified Health Centers, surgical oncology, and medical oncology, and no navigation services in surgical oncology). Perhaps related to the latter, women described radiation as optional, often in the same words as they described breast reconstruction.
\end{abstract}

On behalf of North St. Louis Breast Cancer Mortality Reduction Executive Committee.

Sarah Gehlert

sgehlert@wustl.edu

1 George Warren Brown School of Social Work, Washington University in St. Louis, One Brookings Drive, Campus Box 1196, St. Louis, MO 63130, USA

2 Department of Surgery, Division of Public Health Sciences, Washington University School of Medicine in St. Louis, 660 S. Euclid Ave., St. Louis, MO 63110, USA
Discontinuous and fragmented patient navigation leads to failure to associate radiation therapy with vital treatment recommendations. Better integrated navigation that continues throughout treatment will increase treatment completion with the potential to improve outcomes in African Americans and decrease the disparity in mortality.

Keywords Cancer - Breast cancer - Radiation therapy Treatment $\cdot$ African-American $\cdot$ Disparities

\section{Introduction}

Breast cancer is the second leading cause of cancer death for American women, with an estimated 232,000 new cases of invasive breast cancer and 39,000 deaths reported annually [1]. Despite progress in the detection and treatment of breast cancer, racial/ethnic disparities persist. African-American women have a $41 \%$ greater chance of dying from breast cancer than white women, although white women have higher incidence of the disease [1]. These differences in mortality have been attributed to a number of factors, including problems with accessing treatment due to socioeconomic differences, differences in tumor stage and grade at the time of diagnosis, and variation in tumor biology [2-10].

Breast cancer rates for African-American women in the St. Louis area (29.4 per 100,000 city, 35.7 per 100,000 county) are higher than for the state of Missouri (24.2 per $100,000)$ and the United States $(22.6$ per 100,000) compared to all other races [11]. North St. Louis, a predominantly urban, African-American area, exhibits the lowest life expectancy at birth and the highest cancer mortality in the St. Louis region [12]. Local researchers are focused on assessing and intervening to reduce these trends. Among 
significant findings is that, when compared to women referred from private healthcare facilities, women referred from the St. Louis Safety Net system present with larger breast tumors and experience significant delays in referral to a St. Louis comprehensive cancer center [13, 14].

In attempting to understand contributors to AfricanAmerican and white cancer disparities, investigators have identified factors at multiple levels of influence, from the biological to the societal. These include proximal factors such as biologic/genetic pathways and individual risk factors such as diet, distal factors such as societal norms, and the intermediate factors through which distal factors are experienced, such as social networks and healthcare institutions [15]. All of these pathways and their interactions contribute to breast cancer disparities in the United States. These disparities occur at three primary points: (1) lifetime exposures and stressors; (2) access to health care; and (3) quality of health care received [16]. Few researchers have investigated provider-level barriers to the completion of breast cancer treatment among racial and ethnic minority groups as a contributor to African-American and white breast cancer mortality disparities to the same extent as they have individual-level barriers. An array of providerlevel structural and systemic factors has been suggested (location of facilities, availability of oncology specialists, etc.), but not fully explored [17-22].

This study is guided by the hypothesis that poorly done intra- and inter-organizational referrals for North St. Louis women contribute to their inability to complete prescribed breast cancer treatment and thus to the African-American and white breast cancer mortality disparity. Nationally, African-American women are less likely to complete treatment for breast cancer than white women [7, 23], and we posited that the fragmented way that services are provided and the poor connection between the safety net system and comprehensive cancer center in the region may contribute. We pursued our inquiry using four approaches, as outlined below.

\section{Methods}

\section{Sample}

The primary sample was breast cancer survivors who received their first diagnosis from 2000 to 2008, while living in one of eight zip codes. This concentration of eight St. Louis City zip codes was chosen based on its high rates of late stage at diagnosis forming a North St. Louis cluster of seven zip codes in St. Louis City, an independent entity, and one was in St. Louis County [24]. Among those women in the primary sample that were still alive in 2012, we sampled 96 to participate in qualitative interviews.
We also conducted two focus groups of the St. Louis breast health workforce; participants included breast cancer navigators who participate in the quarterly St. Louis Regional Breast Cancer Workgroup and providers from the federally qualified health center (FQHC) that is a partner in the study and the site at which most women in the Show Me Healthy Women Missouri (SMHW) program receive a certain or probable diagnosis of breast cancer. Clinic providers from the $\mathrm{FQHC}$ and two hospitals that treat the majority of SMHW patients were invited using letters distributed by the partners from their organizations. The Human Research Protections Office of the School of Medicine approved this study.

\section{Data collection}

A mixed methods approach involving both qualitative and quantitative data collection was used to address the study's research question. Due to the lack of understanding of how provider-level factors influence inability to complete treatment, we chose an approach that would allow us to triangulate, or cross verify information using two or more methods. We used four methods: (1) analysis of Missouri Cancer Registry (MCR) data; (2) qualitative interviews with women from the registry who were alive at the time of the study; (3) analysis of electronic medical record data of a subsample of those survivors; and (4) focus group interviews with providers of services to women in the SMHW program.

Quantitative approaches were utilized to examine MCR data to determine who among the women diagnosed with breast cancer while living in the target area during the targeted years was still living and to examine their sociodemographic characteristics and treatment patterns. Data collected from the MCR included year of diagnosis, zip code at diagnosis, type of insurance, tumor characteristics (stage and grade), cancer status (evidence of cancer at last contact), and vital status (whether the women were alive at last contact). The private insurance category included managed care, HMO, PPO, and TRICARE, while the public insurance category included Medicaid and Medicare (with or without supplements) and Veterans Affairs insurance.

Qualitative approaches included interviews with African-American breast cancer survivors from the primary sample. Recruitment was done through invitations sent by the MCR to all women diagnosed during that period while living in the eight zip codes, using their updated list of survivors. Because the vast majority of women in the sample were of very low socioeconomic status, we chose to recruit using the three approaches, anticipating, for example, that women with frequent moves might not receive letters sent by the MCR. We used a multifaceted approach 
supplementing mailed letters with passing out flyers in the eight zip codes and invitations to their patients from our provider partners.

These 60-90 min interviews took place in women's homes and were designed, based on narrative theory, to evoke narratives about each woman's breast cancer treatment experience. They were conducted by two specially trained female African-American interviewers using a guide jointly prepared by the authors and breast cancer survivors living in the same zip codes [25]. Narrative theory holds that narrative is a basic human strategy for coming to terms with fundamental elements of experience, such as time and process. Kleinman [26] has shown that illness is shaped by cultural, social, and environmental factors that affect a person's experience of disease. The stories patients tell provide a means of confronting contradictions between experience and expectations based on shared cultural models about illness and treatment [27].

Among women who participated in the semi-structured interviews, we extracted information from the electronic medical records (EMR) of those who received their treatment at the two primary hospital providers of treatment to women in the study. This was done to obtain what could be gleaned from their treatment histories from the provider perspective, looking especially for evidence of conversations between women and providers. This allowed us to analyze the treatment trajectories of the women interviewed and compare them to the narrative accounts they provided. Our principle aim was to determine the extent to which these women were included in their own care, a major goal of the Affordable Care Act (ACA) [28]. As such, we examined medical charts for provider:patient discussions and treatment completed. We compared the information women reported with that recorded in their EMR to determine concordance. Although we were aware of no standard for recording patient and provider conversations, we wanted to determine if evidence could be found in the EMRs of women in our sample. Consent was obtained in person from each participant in writing prior to each in home interview; this also included consent to extract EMR information.

Finally, we conducted focus groups of patient navigators and provider staff members to gain their perspectives on the breast cancer treatment of women in the North St. Louis cluster. Our provider partners, members of the North St. Louis Breast Cancer Mortality Reduction Executive Committee who represent two hospitals affiliated with an NCI-designated comprehensive cancer center [25], recruited the breast cancer navigators and clinic service providers to participate in the focus groups. Oncology services at the two hospitals share practitioners. The 60-minute focus groups of 8-10 persons were held at a community location and led by the senior author using an interview guide prepared for the study. The interview guide queried participants about how services were delivered to women, what obstacles were perceived, and how navigators and other providers engaged with providers at other sites when making patient referrals.

\section{Analysis}

\section{Examination of MCR data}

Data were examined to determine the number of AfricanAmerican women diagnosed with breast cancer in 2000-2008 while living in one of the eight zip codes. We determined the number of women alive in 2012, and also examined the percentage treated by study partners (see Fig. 1). Univariate statistics were used to summarize each variable taken from the MCR with means, standard deviations, frequencies, and percentages, as appropriate. Given the amount of unknown and missing information leading to small cell counts when stratified, nonparametric Fisher's exact tests were used to determine whether there were bivariate associations between demographic characteristics and clinical, treatment, and outcome variables measured at last contact and categorized as follows: age $(\leq 50, \geq 51)$, insurance type (public, private), cancer status (evidence of tumor, no evidence of tumor), cancer grade (I, II, III, IV),

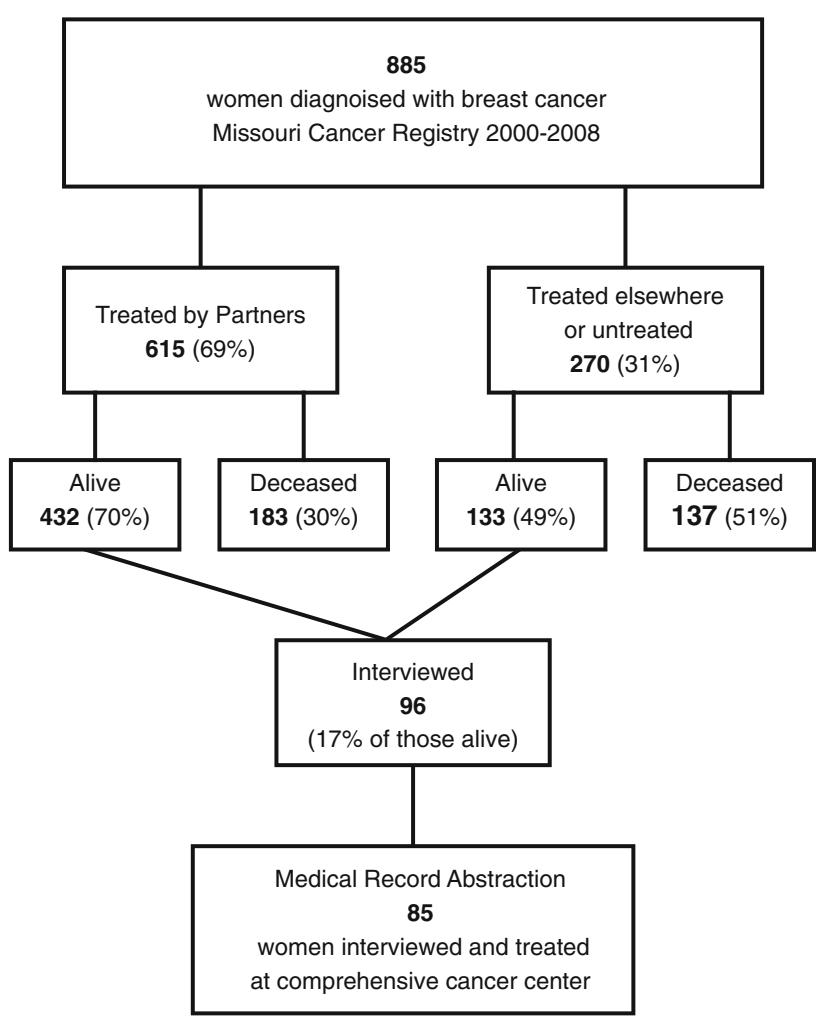

Fig. 1 Determination of women eligible for the study and treated by partners 
surgery recommended as first course of treatment (yes, no), surgery performed as first course of treatment (surgery performed, surgery not part of planned treatment, surgery recommended but not performed), and vital status (alive, dead).

\section{Examination of interview data}

The narrative method of qualitative research was chosen to analyze interview data. As described by Riessman [29], narrative research analysis interprets text from participant stories, paying particular attention to the chronological order of the information. Our approach was to interview and gather the personal experiences of a set of participants and retell their story based on narrative features such as setting, characters, and resolutions [30]. The authors collected the treatment practices of African-American women diagnosed with breast cancer in 2000-2008 while living in the cluster of zip codes. Interviews began with a discussion of the participant's understanding of her prescribed treatment and moved through events with her treatment experience. Key points were then mapped onto a timeline to allow comparison across participants.

Interviews were transcribed, checked for accuracy, and used to create an analytic narrative for each participant. Analytic narratives were aggregated according to the treatment experiences of breast cancer patients (e.g., screening and diagnosis, surgery, treatment, support/barriers) and compared across participants. After interview data were synthesized, themes were developed according to similarities of content across participants, and these themes were reviewed independently for completeness by the first and senior authors. NVivo 10.0 was used to store and analyze all qualitative data files.

\section{Examination of EMR data}

Participant data were extracted from electronic medical record (EMR) programs used at the academic medical system: allscripts, used by outpatient clinics, and ClinDesk, used by inpatient clinics. The EMRs were searched for individual characteristics (age, income at time of diagnosis), clinical characteristics (tumor histology, stage, grade, hormone receptor status), and curative breast cancer treatment characteristics (modalities, start dates, end dates, explanations of treatment decisions, and/or delays) [31]. The types of EMR documents analyzed included pathology reports, treatment consultations, physician/nurse notes, operative summaries, and treatment summaries. When a chart entry indicated that a provider had conversed with a participant about treatment, treatment information was recorded as having been discussed.

\section{Results}

Analysis of Missouri Cancer Registry data identified a total of 885 African-American women who were diagnosed with breast cancer from 2000 to 2008 while residing in one of the eight target zip codes. The mean age was 61 years $(M=60, S D=15)$ at the time of the study, and 565 $(64 \%)$ of the 885 women were living (Table 1). The majority of women were recommended $91 \%(n=770)$ and received $84 \%(n=742)$ surgery as a first course treatment (Table 2). Bivariate analysis revealed that age was significantly associated with receipt of first course surgery $(p=0.007)$, cancer grade $(p=0.003)$, and vital status $(p<0.001)$. Insurance status was significantly associated with vital status $(p=0.009)$, surgery recommended ( $p<0.001)$, and surgery performed $(p<0.001)$ as first course of treatment and cancer grade $(p=0.024)$. We found that women who did not receive surgery as a first course treatment tended to be older, have public insurance, less likely to have localized tumors, and had tumors that were larger.

Ninety-six (17\%) of the 565 eligible women alive at the time of the study agreed to be interviewed for the study. They had a mean age of 63 years. Half received their suspicious or confirmed diagnosis of breast cancer through a screening mammogram at an FQHC, St. Louis County clinic, community clinic, or hospital. Eighty-five women $(89 \%)$ eventually received treatment at the NCI-designated cancer center with which the two hospitals are affiliated.

Medical record extractions were completed for all 85 participants. The EMRs were examined to determine what treatment information or instruction was given to the women by providers, identify the services they received, and compare with interview data.

The 96 interviews analyzed yielded two overarching themes: (1) fragmentation of services and (2) limited knowledge of breast cancer treatment. First, the fragmentation of services for women led to increased delays in treatment initiation. Half $(n=48)$ of the participants reported receiving an abnormal mammogram at a community hospital or FQHC. Thirteen of those women reported completing appointments at a second community site before connecting to a treatment facility, adding a delay in treatment initiation.

Second, participants said that they had difficulty processing treatment information due to nerves and the speed with which it was presented to them. One participant reported, "[They] wanted me to have surgery... I thought that I should get a second opinion because I didn't understand why me. But I did not know how." In particular, women reported problems understanding the purpose 
Table 1 Selected characteristics of AfricanAmerican breast cancer cases diagnosed in the in the eight target zip codes between 2000 and 2008

\begin{tabular}{|c|c|c|}
\hline Variable $(n=885)$ & Frequency & Percent \\
\hline \multicolumn{3}{|l|}{ Demographic characteristics } \\
\hline Age at diagnosis: mean (SD) & $61(15)$ & \\
\hline \multicolumn{3}{|l|}{ Age category } \\
\hline$\leq 50$ & 254 & 29 \\
\hline$\geq 51$ & 631 & 71 \\
\hline \multicolumn{3}{|l|}{ County of residence at diagnosis } \\
\hline St. Louis County & 620 & 70 \\
\hline St. Louis City County & 265 & 30 \\
\hline \multicolumn{3}{|l|}{ Type of insurance ${ }^{a}$} \\
\hline No insurance & 1 & 0.1 \\
\hline Public insurance & 160 & 18 \\
\hline Private insurance & 78 & 39 \\
\hline Unknown/missing & 646 & 73 \\
\hline \multicolumn{3}{|l|}{ Clinical characteristics } \\
\hline \multicolumn{3}{|l|}{ Cancer stage } \\
\hline Localized & 219 & 25 \\
\hline Regional & 171 & 19 \\
\hline Distant & 41 & 5 \\
\hline Unknown/missing & 454 & 51 \\
\hline \multicolumn{3}{|l|}{ Cancer grade } \\
\hline Grade I & 128 & 14 \\
\hline Grade II & 266 & 30 \\
\hline Grade III & 380 & 43 \\
\hline Grade IV & 2 & 0.2 \\
\hline Unknown/missing & 109 & 12 \\
\hline \multicolumn{3}{|l|}{ Treatment characteristics } \\
\hline \multicolumn{3}{|l|}{ Surgery recommended as first course treatment } \\
\hline Yes & 770 & 87 \\
\hline No & 79 & 9 \\
\hline Unknown & 36 & 4 \\
\hline \multicolumn{3}{|l|}{ Surgery was performed as first course treatment } \\
\hline Surgery was performed & 742 & 84 \\
\hline Surgery not part of planned first course treatment & 79 & 9 \\
\hline Surgery was recommended but not performed & 26 & 3 \\
\hline Unknown & 38 & 4 \\
\hline \multicolumn{3}{|l|}{ Outcomes } \\
\hline \multicolumn{3}{|l|}{ Cancer status at last contact } \\
\hline No evidence of tumor & 614 & 69 \\
\hline Evidence of tumor & 217 & 25 \\
\hline Unknown & 54 & 6 \\
\hline \multicolumn{3}{|l|}{ Vital status at last contact } \\
\hline Patient alive & 565 & 64 \\
\hline Patient deceased & 320 & 36 \\
\hline
\end{tabular}

a Public insurance included: Medicaid (includes those managed through a manage care plan), Medicare (with and without supplements), Veteran Affairs, private insurance included: managed care plans, preferred provider organizations (PPOs), health maintenance organizations (HMOs), Tricare of radiation therapy. When asked "Did you understand why they wanted you to have radiation before surgery?" one woman replied, "They wanted to shrink it first. But I had done some research on radiation and I found out that was not successful even though people went through that. People did not recover. I did not want them to experiment 
Table 2 Significant associations between demographic, clinical, treatment, and outcome variables of African-American women diagnosed between 2000 and 2008 while residing in the eight target zip codes

\begin{tabular}{|c|c|c|}
\hline Demographic/clinical variables & Clinical/treatment/outcome variables & $p$ value* \\
\hline Age $(\leq 50, \geq 51)$ & Surgery performed $* *$ & $p=0.007$ \\
\hline Age & Alive at last contact & $p=0.003$ \\
\hline Age & Cancer grade & $p<0.001$ \\
\hline Insurance type (public, private) & Alive at last contact & $p=0.009$ \\
\hline Insurance type & Surgery recommended & $p<0.001$ \\
\hline Insurance type & Surgery performed $* *$ & $p<0.001$ \\
\hline Insurance type & Cancer grade & $p=0.024$ \\
\hline Cancer stage $* * *$ & Surgery recommended & $p<0.001$ \\
\hline Cancer stage $* * *$ & Surgery performed $* *$ & $p<0.001$ \\
\hline Cancer stage $* * *$ & Alive at last contact & $p<0.001$ \\
\hline Cancer stage $* * *$ & Evidence of tumor at last contact & $p<0.001$ \\
\hline Cancer grade & Alive at last contact & $p<0.001$ \\
\hline
\end{tabular}

* Fisher's exact test

** Surgery performed as first course of treatment categorized (surgery performed, surgery not part of planned treatment, surgery recommended but not performed)

*** Cancer stage is categorized (localized, regional, distant) on me." Another participant expressed concern about the side effects of radiation treatment, stating, "I was afraid because I lost my grandmother to cancer that I heard many things about cancer, negative things. I was angry and did not want to go through the side effects of radiation. I finally broke down when....I finally decided to do it when they told me about the success rates of survival."

Review of navigator and provider focus group transcripts yielded similar themes. Focus group members echoed the theme of service fragmentation. The system of linking FQHC breast navigators with patients involved telephone calls rather than on-site introductions. Navigators reported receiving women's contact information by a primary care physician after the women received a confirmed or probable diagnosis, yet in many cases, by the time that they called, the contact information was no longer valid. Navigators reported frustration in trying to deal with women's emotions by phone. This was particularly acute in cases where the women were frightened at the beginning of treatment or on the verge of dropping out. Navigators relayed that while they communicate with one another in quarterly St. Louis Regional Breast Cancer Workgroup meetings, ensuring continuity of care between sites and settings remains problematical.

In comparing EMR entries with patient narratives, we noted a disconnection between what women described as recommended or optional treatments and what physicians recorded in the EMR. For example, one physician recorded, "A 48 year old lady with invasive ductal carcinoma with DCIS of left breast, which is triple negative underwent partial mastectomy and had radiation oncology consultation. I also [stated] that she seriously reconsider chemotherapy as she is perimenopausal with a hormone receptor negative tumor greater than $1 \mathrm{~cm}$." The patient reported that she declined chemotherapy and was unable to relate the information conveyed to her in any detail.

\section{Discussion}

Fragmentation of services emerged as a major obstacle to treatment for women living in our area of focus in North St. Louis that emerged from both narrative interviews with women and focus groups of providers. Having navigators associated with individual services (surgical, medical, and radiation oncology), and settings (the FQHC and individual hospital clinics) presents an enormous challenge to the continuity of care that is essential to ensuring that lower SES women engage in and are able to complete breast cancer treatment. While communicating regularly with one another through quarterly meetings of navigators may help to contribute to women completing prescribed treatment, thus decreasing the mortality disparity, it is not enough. Our findings suggest that tying navigators to episodes of breast cancer rather than services is a better alternative, very much in keeping with tenets of the ACA [28].

Additionally, radiation oncology was the service least understood by women in the study in terms of its connection to survival. Chart review revealed that patient navigation tapers off after surgery, with only one navigator employed by medical oncology and none in radiation oncology. We likewise found no evidence that interprofessional team meetings included navigators. Our findings support previous research showing the failure to ensure continuity of service between radiation oncology and medical oncology and surgery may interfere with the receipt of services [31]. In comparing the women's stories about treatment and that of the attending physicians as 
recorded in the patient records, we found only two instances in which providers recorded conversations with women, and neither indicated shared decision making. Although this may be due to conventions of recording conversations with patients among oncologists at this cancer center, it fits with what investigators like Peek et al. have found about disparities in shared decision making between African and Americans with diabetes and their providers [32]. We did find a strong temporal tie between women's understanding of a treatment's link to survival and whether a navigator was available on the service. Women were more likely to perceive radiation therapy as optional rather than linking it to survival than chemotherapy and surgery. Where study participants appeared hesitant to follow through on chemotherapy, they often did so based on encouragement from patient navigators. That opportunity was not available for patients facing radiation therapy.

The woman's description of treatment during the interview did not always match what was recorded in the medical record, compounded by an almost total lack of recording about communication with women about their treatment. We were able to determine, however, that while some women expressed feeling that radiation was a more optional aspect of breast cancer treatment, according to the medical records, approximately $60 \%$ of the women received radiation therapy. This observation may have been the result of difficulty remembering treatment consultations or treatments received, misunderstanding the difference between radiation and chemotherapy, or could have reflected their thoughts about treatment regardless of whether treatment was actually initiated. This may also suggest that because of lack of knowledge about breast cancer treatment, many women still default to their providers to make treatment decisions. It also may stem from a failure of providers to discuss treatment with women whom they assume will either not be able to understand their explanations or are less interested in their care, as suggested by the Institute of Medicine report, Unequal Treatment: Confronting Racial and Ethnic Disparities in Health Care [33].

The analysis of data from the Missouri Cancer Registry allowed us to gain a broader view of breast cancer treatment patterns in African-American women who reside in North St. Louis. Although this analysis did not directly relate to our hypothesis, it was necessary as a first step to characterize the data and to identify women to interview. It therefore informed the rest of the study. Approximately $12 \%$ of the women in this sample did not receive surgery as a first course of treatment. Differences in demographic and clinical characteristics may shed some light on this observation. Statistical analysis suggests that the age and insurance status of this subsample of women may have contributed to their relatively higher death rates. Women who did not receive surgery as first course treatment were older, more likely to have public insurance, and had larger tumors, fewer of which were localized. It is likely the larger tumors and more advanced tumor stages required neoadjuvant chemotherapy to shrink the tumors before surgery or subsequent adjuvant therapies. Due to the lack of data on treatment beyond first course, we were unable to assess whether women with neoadjuvant chemotherapy received surgery at a later time or whether women received appropriate adjuvant therapies.

These women also had lower survival rates $(30 \%)$ compared to those with first course surgical treatment $(70 \%)$. The reduced survival may have resulted from their older ages; older women are more likely to die from breast cancer [34-37]. Interestingly, mortality rates for older women have not improved as much as those for younger women [35]. This reduced survival may be due to undertreatment resulting from concerns about comorbidities and treatment toxicity, or age bias on the part of the physician $[35,38,39]$. Older women are less likely to receive aggressive surgical treatment, systematic treatment $[35,38,40]$, or expected and concordant treatment for breast cancer [39, 40]. Additionally, African-American women, those with public insurance, and those living in impoverished, urban areas are also less likely to receive expected and concordant care [41, 42]. The majority of the women in this sample had all of these characteristics.

Acknowledgments This work is supported by Susan G. Komen for the Cure $^{\circledR}$ Grant SG-11-10005, NCI U54-153460, CTSA UL1RR024992, U54 CA153460, and the Barnes-Jewish Foundation. We would like to thank the Executive Committee and community partners for "A Community-Based Approach to Improving Breast Cancer Services for African American Women Living in St. Louis." We would also like to thank the Missouri Cancer Registry staff and the breast cancer survivors who participated in this study.

\section{Compliance with ethical standards}

Conflict of Interest The authors declare that they do not have any conflicts of interest.

Open Access This article is distributed under the terms of the Creative Commons Attribution-NonCommercial 4.0 International License (http://creativecommons.org/licenses/by-nc/4.0/), which permits any noncommercial use, distribution, and reproduction in any medium, provided you give appropriate credit to the original author(s) and the source, provide a link to the Creative Commons license, and indicate if changes were made.

\section{References}

1. DeSantis C, Ma J, Bryan L, Jemal A (2014) Breast cancer statistics, 2013. CA Cancer J Clin 64:52-62

2. Carey LA, Perou CM, Livasy CA et al (2006) Race, breast cancer subtypes, and survival in the Carolina breast cancer study. JAMA 295:2492-2502 
3. van Ravesteyn NT, Schechter CB, Near AM et al (2011) Racespecific impact of natural history, mammography screening, and adjuvant treatment on breast cancer mortality rates in the United States. Cancer Epidemiol Biomark Prev 20:112-122

4. Vona-Davis L, Rose DP (2009) The influence of socioeconomic disparities on breast cancer tumor biology and prognosis: a review. J Womens Health 18:883-893

5. Barry J, Breen N, Barrett M (2012) Significance of increasing poverty levels for determining late-stage breast cancer diagnosis in 1990 and 2000. J Urban Health 89:614-627

6. DeSantis C, Naishadham D, Jemal A (2013) Cancer statistics for African-Americans, 2013. CA Cancer J Clin 63:151-166

7. Fedewa SA, Edge SB, Stewart AK, Halpern MT, Marlow NM, Ward EM (2011) Race and ethnicity are associated with delays in breast cancer treatment (2003-2006). J Health Care Poor Underserved 22:128-141

8. Kim S, Chukwudozie B, Calhoun E (2013) Sociodemographic characteristics, distance to the clinic, and breast cancer screening results. J Health Dispar Res Pract 6:70

9. Akinyemiju T, Soliman A, Johnson $\mathrm{N}$ et al (2013) Individual and neighborhood socioeconomic status and healthcare resources in relation to black-white breast cancer survival disparities. J Cancer Epidemiol 1:1-13

10. Campbell RT, Li X, Dolecek TA, Barrett RE, Weaver KE, Warnecke RB (2009) Economic, racial and ethnic disparities in breast cancer in the US: towards a more comprehensive model. Health Place 15:855-864

11. State Cancer Profiles. http://statecancerprofiles.cancer.gov/. Accessed March 232015

12. City of St. Louis Department of Health. Public health: Understanding our needs. http://stlouis-mo.gov/government/depart ments/health/documents/public-health-understanding-our-needsreport.cfm. Published July, 2011, Retrieved March 232015

13. Fayanju OM, Jeffe DB, Elmore L, Ksiazek DN, Margenthaler JA (2013) Patient and process factors associated with late-stage breast cancer diagnosis in safety-net patients: a pilot prospective study. Ann Surg Oncol 20:723-732

14. Fayanju OM, Jeffe DB, Tappenden JR et al (2012) Breast cancer presentation in an urban health care safety net system. Mo Med 109:405-411

15. Holmes JH, Lehman A, Hade E, Ferketich AK, Gehlert S, Rauscher GH, Abrams J, Bird CE (2008) Challenges for multilevel health disparities research in a transdisciplinary environment. Am J Prev Med 35(2):S182-S192

16. Jones CP, Jones CY, Perry GS, Barclay G, Jones CA (2009) Addressing the social determinants of children's health: a cliff analogy. J Health Care Poor Underserved 20(4A):1-12

17. Bickell NA, McEvoy MD (2003) Physicians' reasons for failing to deliver effective breast cancer care: a framework for underuse. Med Care 41:442-446

18. Demissie S, Silliman RA, Lash TL (2001) Adjuvant tamoxifen: predictors of use, side effects, and discontinuation in older women. J Clin Oncol 19:322-328

19. Hershman DL, Shao T, Kushi LH et al (2011) Early discontinuation and non-adherence to adjuvant hormonal therapy are associated with increased mortality in women with breast cancer. Breast Cancer Res Treat 126:529-537

20. Magai C, Consedine N, Neugut AI, Hershman DL (2007) Common psychosocial factors underlying breast cancer screening and breast cancer treatment adherence: a conceptual review and synthesis. J Womens Health 16:11-23

21. Penner LA, Eggly S, Griggs JJ, Underwood W, Orom H, Albrecht TL (2012) Life-threatening disparities: the treatment of black and white cancer patients. J Soc Issues 68(2):328-357

22. Wheeler SB, Reeder-Hayes KE, Carey LA (2013) Disparities in breast cancer treatment and outcomes: biological, social, and health system determinants and opportunities for research. Oncologist 18:986-993

23. Curtis E, Quale C, Haggstrom D, Smith-Bindman R (2008) Racial and ethnic differences in breast cancer survival: how much is explained by screening, tumor severity, biology, treatment, comorbidities, and demographics? Cancer 112:171-180

24. Lian M, Jeffe DB, Schootman M (2008) Racial and geographic differences in mammography screening in St. Louis City: a multilevel study. J Urban Health 85(5):677-692

25. Gehlert S, Fayanju OM, Jackson S et al (2014) A method for achieving reciprocity of funding in community-based participatory research. Prog Community Health Partnersh 8(4):561-570

26. Kleinman A (1988) The illness narratives: suffering, healing, and the human condition. Basic Books, New York

27. Mattingly C, Garro LC (2000) Narrative and the cultural construction of illness and healing. University of California Press, Berkeley

28. Patient Protection and Affordable Care Act. P.L. 111-148, 124 Stat. 119. http://www.gpo.gov/fdsys/pkg/PLAW-111publ148/pdf/ PLAW-111publ148.pdf. Accessed March 232015

29. Riessman CK (2008) Narrative methods for the human sciences. Sage, Thousand Oaks

30. Creswell JW (2013) Research design: qualitative, quantitative, and mixed methods approaches. Sage, Thousand Oaks

31. Connors SK, Goodman MS, Noel L et al (2014) Breast cancer treatment among African American women in North St. Louis, Missouri. J Urban Health 92:1-16

32. Peek ME, Odoms-Young A, Quinn MT et al (2010) Race and shared decision-making: perspectives of African-Americans with diabetes. Soc Sci Med 71(1):1-9

33. Shen MJ, Binz-Scharf M, D'Agostino T et al (2015) A mixedmethods examination of communication between oncologists and primary care providers among primary care physicians in underserved communities. Cancer 121:908-915

34. Smedley B, Stith A, Nelson A (eds) (2003) Unequal treatment: confronting racial and ethnic disparities in healthcare. National Academies Press, Washington

35. Smith BD, Jiang J, McLaughlin SS et al (2011) Improvement in breast cancer outcomes over time: are older women missing out? J Clin Oncol 29:4647-4653

36. Yancik R, Ries LG, Yates JW (1989) Breast cancer in aging women. A population-based study of contrasts in stage, surgery, and survival. Cancer 63:976-981

37. Mueller CB, Ames F, Anderson GD (1978) Breast cancer in 3558 women: age as a significant determinant in the rate of dying and causes of death. Surgery 83:123-132

38. Muss HB, Busby-Whitehead J (2011) Older women with breast cancer: slow progress, great opportunity, now is the time. J Clin Oncol 29:4608-4610

39. Greenfield S, Blanco DM, Elashoff RM, Ganz PA (1987) Patterns of care related to age of breast cancer patients. JAMA 257:2766-2770

40. Merchant TE, McCormick B, Yahalom J, Borgen P (1996) The influence of older age on breast cancer treatment decisions and outcome. Int J Radiat Oncol Biol Phys 34:565-570

41. Breen N, Wesley MN, Merrill RM, Johnson K (1999) The relationship of socio-economic status and access to minimum expected therapy among female breast cancer patients in the National Cancer Institute Black-White Cancer Survival Study. Ethn Dis 9(1):111-125

42. Anderson RT, Morris CR, Kimmick G et al (2014) Patterns of locoregional treatment for nonmetastatic breast cancer by patient and health system factors. Cancer 121:790-799 